\title{
Scaling and Adapting a Program for Early Undergraduate Research in Computing
}

\author{
Christine Alvarado \\ cjalvarado@ucsd.edu \\ UC San Diego \\ USA
}

\author{
Joe Hummel \\ jhummel2@uic.edu \\ University of Illinois, Chicago
}

USA

\author{
Diba Mirza \\ dimirza@cs.ucsb.edu \\ UC Santa Barbara \\ USA
}

\author{
Renata Revelo \\ revelo@uic.edu \\ University of Illinois, Chicago
}

USA

\begin{abstract}
The Early Research Scholars Program (ERSP) was launched in 2014 at UC San Diego as a way to provide the benefits of research experiences to a large and diverse group of students early in their undergraduate computing career. ERSP is a structured program in which second-year undergraduate computing majors participate in a group-based, dual-mentored research apprenticeship over a full academic year. In its first four years ERSP engaged 139 students with a high proportion of women $(68 \%)$ and racially minoritized students (19\%), and participation in ERSP correlated with increased class grades. In 2018 we partnered with three additional universities to launch their own version of ERSP. Implementations at our partner sites have seen similar diversity and initial success, and have taught us how to implement the program in different contexts (e.g. quarters vs. semesters, different credit structures). This paper describes the structure of ERSP and how it can be adapted to different contexts to construct a scalable and inclusive research experience for early-career undergraduates in computing and related fields.
\end{abstract}

\section{CCS CONCEPTS}

-Social and professional topics $\rightarrow$ Computing education programs.

\section{KEYWORDS}

Undergraduate Research; Diversity; Early Undergraduates; Scaling Research; Computing

\section{ACM Reference Format:}

Christine Alvarado, Joe Hummel, Diba Mirza, Renata Revelo, and Lisa Yan. 2022. Scaling and Adapting a Program for Early Undergraduate Research in Computing. In Proceedings of the 53rd ACM Technical Symposium on Computer Science Education V. 1 (SIGCSE 2022), March 3-5, 2022, Providence, RI, USA. ACM, New York, NY, USA, 7 pages. https://doi.org/10.1145/3478431. 3499336

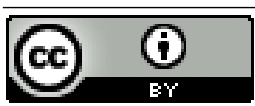

This work is licensed under a Creative Commons Attribution International 4.0 License.

SIGCSE 2022, March 3-5, 2022, Providence, RI, USA

(C) 2022 Copyright held by the owner/author(s).

ACM ISBN 978-1-4503-9070-5/22/03.

https://doi.org/10.1145/3478431.3499336

\author{
Lisa Yan \\ yanlisa@eecs.berkeley.edu \\ UC Berkeley \\ USA
}

\section{INTRODUCTION}

Undergraduate research experiences (UREs) have long been shown to have positive outcomes for students [13,14]. In computing specifically, students in UREs benefit from increased retention [4, 7], increased self-efficacy and scientific identity [11], and improved academic outcomes [4]. Benefits are even more pronounced for students from groups underrepresented in computing, including women and Black, Latinx and Native American students [11, 17]. Additionally, there is evidence that early research engagement may be beneficial for increasing diversity in computing [16].

Yet at most large research universities, undergraduate research opportunities in computing are mostly ad hoc and difficult to obtain, particularly for early undergraduates in their first or second year. Even when faculty are willing to take early undergraduates, it is time-consuming to train students-not only in the knowledge and skills of the specific research area, but also in the process of conducting research. As a result, relatively few early undergraduates have the opportunity to engage in real research.

Recognizing the need for a sustainable undergraduate research pipeline, the Computing Research Association's Education Committee (CRA-E) recently released a report on best practices for building a large-scale research program in computing [3]. However, the task of designing such a program is left to the reader.

This paper presents details of a scalable research program for early undergraduates in computing, called the Early Research Scholars Program (ERSP) [6] (http://ersp.ucsd.edu), that follows the best practices outlined in the CRA-E report. ERSP has been running continuously since 2014 at UC San Diego (UCSD), was launched at three additional universities in 2018 and 2019, and will launch at four more over the next two years. ERSP provides research opportunities for up to 50 students per year (between $5-15 \%$ of the second-year students in computing, depending on the institution), and the majority of ERSP students are from groups that are underrepresented in computing. Our goal with this report is to describe the essential components of ERSP, and the tips and variations we have discovered to make the program successful in a variety of contexts in order to allow others to implement this successful program at their institution.

\section{RELATED WORK}

Undergraduate research has been studied extensively due to its impact on student academic outcomes [9]. UREs vary in their scale, 
structure and support for students, ranging from in-course research opportunities to academic year co-curricular projects, to full-time summer programs. In many cases, a URE resembles a scaled-down graduate research experience, with an undergraduate student working on an individual project under the guidance of a faculty mentor and possibly their graduate student(s). In these situations, the student experience is largely dependent on the quality of the mentoring and the structure that the faculty member puts into the project. Several researchers have studied the traits of effective mentors $[10,15,18,20]$ and found that critical aspects of a successful mentor include professional socialization, intellectual/research support, personal/emotional support, support for diversity, and the ability to give students freedom while controlling the direction and outcomes of the research.

Yet not all mentors possess the above characteristics; moreover, mentorship can affect students differently by race and gender. For example, Barker found that mentors would sometimes display unconscious bias against women, which would consequently negatively impact their (women) research students [5]. Aikens et al found that some research groups (including women, but also White and Asian students) were more likely to receive direct mentoring from only a graduate student and not a faculty member, which resulted in more negative outcomes by retention and identity [1]. In a study of of 117 computing UREs, Rorrer et al. also found differences by gender and race, but with more positive gains for women than men; and Black, Latinx and Native American students than White and Asian students [11].

Because of the variability and challenge of ad-hoc individual faculty research experiences, various programs have been developed to support undergraduate research in computing. The UR STEM program is a summer URE that specifically recruits and admits students at risk of dropping out of the CS program [7], and has been shown to increase retention for these students. The Affinity Research Group model is a supportive research mentoring structure [19], and in 2019 it was used to construct a summer research experience in which participants were paired together and mentored by both a graduate student and a faculty mentor [8]. Ross et al. describes a virtual URE structure in computing education research that was explicitly designed to foster a sense of community and increase interest in CS education research in a virtual setting [12]

The novel contribution of ERSP is not limited to its combination of explicit mentoring and coursework structure, its engagement of second-year undergraduates, and its focus on equity. ERSP also has a uniquely large scale: It is the first structured program in computing that we know of that can accommodate up to 50 second-year undergraduates each year at a single university and that has been implemented at four different universities. ERSP is a robust, versatile model that is reasonably straightforward to implement and can bring high-quality research experiences to a large number of students who otherwise would not have access to these opportunities.

\section{ESSENTIAL PROGRAM COMPONENTS}

ERSP has two primary goals: (1) to connect second-year undergraduates (particularly those from traditionally minoritized backgrounds) with cutting-edge computing research projects; and (2) to create a inclusive and supportive community around research in computing.

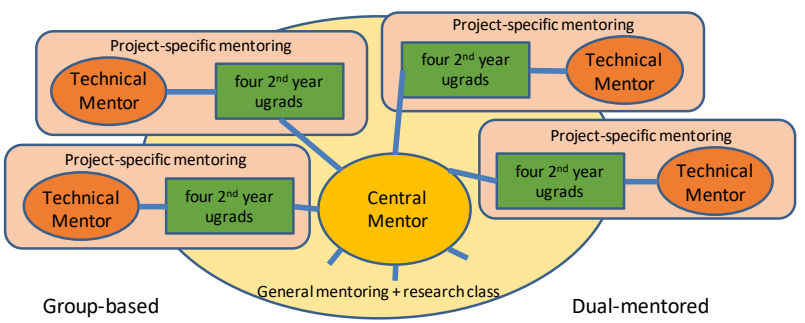

Figure 1: Basic ERSP group-based research and dual mentor structure.

Here we briefly highlight the critical components of ERSP that help achieve these goals. We give more details of the implementation, including variations by institution, in the next section.

Research Course. All students in ERSP first take a formal course, Introduction to Research. The course introduces students to key research practices, such as literature review and oral and written communication, in the context of their assigned project. The course's final deliverable is a formal proposal for a research project that students carry out over the remainder of the ERSP program.

Group-based research. Students in ERSP are grouped into groups of 2-4 students at the start of the program and perform research with this group over the full program.

Dual Mentoring. Students in ERSP have two mentors (Figure 1). A graduate student central mentor meets weekly with every ERSP team to provide general mentoring support such as goal setting and basic technical support. Each team is also assigned to a projectspecific research advisor, or technical mentor (a faculty member, postdoc, graduate student, or combination of these) who meets weekly with the project team to provide more in-depth technical support. This dual-mentoring structure provides benefits to both the ERSP participants and their technical mentors [2].

Program Timeline. ERSP typically runs during the academic year. Students are accepted into the program in the spring or summer of their first year, then grouped and matched with their technical mentors just before the start of the fall term. They take the Introduction to Research course in the fall and carry out their proposed research project over the winter and spring, ending with a poster presentation at the end of the spring term. The academic-year timeline allows students to accept industry (or research) internships or take courses during the summer.

Student population. Students in ERSP are disproportionately members of groups that are underrepresented in computing research. ERSP uses a selection criteria (described in Section 4.7) designed to foster equity and diversity and to develop a large and supportive community of early undergraduate researchers.

\section{IMPLEMENTATION DETAILS}

UCSD launched ERSP in 2014; since 2018, three additional schoolsUC Santa Barbara (UCSB), University of Illinois, Chicago (UIC), and Stanford-have launched ERSP implementations. Each implementation shares the core elements of the program: an introductory research course, dual-mentoring, group-based research, and a focus on diversity and inclusion. However, differences in implementation have taught us about what works well in a variety of contexts. 
In this section we provide more details of ERSP implementation, highlighting similarities and difference across the four institutions. All of the resources discussed in this section (rubrics, course materials, etc.) are available in a startup guide on the ERSP website: https://ersp.eng.ucsd.edu/resources/startup-course-materials.

\subsection{University Context}

ERSP is designed to be implemented in research-intensive universities where there are active research projects but where early undergraduates may not have access to participate. All current partner institutions are research-active doctoral granting institutions in the United States, but they vary in their size, selectivity and structure. UCSD, UCSB, and UIC are all public institutions; Stanford is private. UCSD, UCSB and Stanford are highly selective institutions; UIC is somewhat less selective. UCSB and UIC are classified as Hispanic Serving Institutions, though the participation of Latinx students in computing is relatively low (25\% or less). UCSD's CS department is very large (over 1600 majors); the other partners' departments are moderately sized (400-800 majors). Finally, UCSD, UCSB and Stanford operate on a quarter-system (three 10-week quarters per academic year); UIC uses semesters.

\subsection{Timeline Variations}

Most ERSP programs (UCSD, UCSB and UIC, as well as new partners launching in Fall 2021) follow the timeline structure described in Section 3, where the Introduction to Research course is offered in fall and students complete their proposed projects in winter and spring. Yet, it is possible to offer ERSP in a more compact timeline, and/or to use it as a bridge to (existing) summer research programs. Due to course and staff scheduling constraints, Stanford has implemented two different programs that span just one academic quarter but include both a formal research methods course and an in-depth research experience. All students take the Introduction to Research course, but only students participating in the department's existing summer URE continue after this first quarter. To ensure that the one-quarter students get an authentic research experience, Stanford runs a group-based program where a graduate TA is both the technical and central mentor for each group research project. The students in the summer URE perform individual research. In the spring quarter while they are taking the course they are dual-mentored by their summer URE technical mentor and the central mentor, and mentored by just their technical research mentor through the summer. These two versions were designed not only to address the lack of peer support and community mentoring in Stanford's existing, competitive department URE, but also to create an academic-year research opportunity for more students.

\subsection{Credit Structures and Program Staff}

Students in ERSP receive academic credit (and not a monetary stipend) for their participation. The amount of credit and how ERSP counts towards students' degrees varies across implementations. At UCSD, UCSB, and Stanford, the Introduction to Research course counts as one upper division major elective. Additionally at UCSD and UCSB, students earn 2 research units in each of winter and spring, which can be combined to count as a second major elective. At UIC, which is semester-based, the Introduction to Research course is a 1-credit course taught in fall, followed in spring by $2-3$ credits. UIC hosts students from two departments: Computer Science (CS) and Electrical and Computer Engineering (ECE). Students in both departments earn 3 research credits toward graduation, but these credits only count as elective credit in the major in CS.

The key personnel necessary to launch ERSP are a faculty director, a central mentor, and technical mentors. The faculty director is usually a faculty member in the department of launch; they are in charge of overseeing the program, hiring personnel, and teaching the Introduction to Research course. The faculty director typically receives teaching credit, though some partners have had to initially teach the course as an overload. In addition, faculty directors typically receive summer salary for program coordination. The central mentor (graduate student) receives a stipend for their work throughout the program, with the amount depending on the size of the program and how many hours they work. At UCSD and Stanford, the central mentor works 20 hours/week to mentor between 7 (Stanford) to 14 (UCSD) project groups. At UCSB and UIC, the central mentor works 10 hours/week to mentor 8 projects, though UCSB is currently expanding their program and will support the central mentor at 20 hours/week going forward. Finally, technical mentors (faculty, postdocs, graduate students) are not financially compensated. Despite the fact that they are not paid, we have found that many faculty are eager to participate in ERSP for the incentive of working with well-supported undergraduates-a number of whom continue to contribute productively in their research groups after ERSP ends.

\subsection{Course Content}

The Introduction to Research course is a critical component of the ERSP structure. UCSD and UCSB run a 10-week, 4-unit course that meets twice per week for 80 minutes each session. UIC and Stanford have courses that meet formally only once per week for 50 minutes each session; UIC's course is 15 weeks and 1-unit, while Stanford's course is 10 weeks and 3 or 4 units, depending on which track students are in (see Section 4.2). In all implementations, students attend at least two additional meetings with technical or central mentors, for about 3-4 hours total course meeting time per week.

Each of the four courses covers similar topics and assignments including the following: What is Research?, Teamwork, Reading a Research Paper, Literature Searching, Setting Research Direction and Goals, Experimentation and Evaluation, Written and Oral Communication, Peer Review, Research Career Paths, and Ethics. The major course deliverables are a written project proposal containing a related work section and a proposed execution plan, and a final oral presentation of the research proposal. Students also document weekly logs on their research progress, which are shared with both central and technical mentors. Despite the longer semester schedule, the 1-unit course load at UIC constrains the amount of work that students can do in the fall.

Instead of teaching students the specific knowledge they will need to perform their research project, the course instead teaches meta-skills of research that students, guided by the course instructor and technical mentor, can then apply to teach themselves what they need. A critical aspect of the course is that students immediately apply these meta-skills to their research. For example, when 
students learn to read a research paper, they first read a single shared research paper in a guided in-class activity. Then in their homework assignment, students apply the skills learned in class to read a paper selected by their research mentor that relates directly to their specific project.

\subsection{Project Selection and Group Formation}

ERSP research projects need to be appropriately scoped for undergraduates with only basic computing (or Electrical Engineering) knowledge, yet still part of a real research project. We have found that the best projects are those where students can achieve some results relatively quickly, but then may have to learn more to accomplish the main piece of the project. For example, a machine learning project might begin with testing simple models using off-the-shelf tools before developing more sophisticated techniques.

To ensure successful matching between students and projects/ mentors, we have found the following approach to be successful. Prior to the program's start, the ERSP directors contact the interested technical mentors for the following: a short project description, the knowledge and skills that students will have to acquire to be successful in the project, and the research group's weekly meeting time. This information is then distributed to accepted ERSP students, who rank their interest in the projects (very/somewhat/not at all interested) and also indicate their availability during the group's research meeting times. ERSP directors then match students with projects: Students must be matched with a project whose group meeting they can attend; students should not be matched with a project in which they are not at all interested (and they should be matched with a project that they are very interested in whenever possible); and there should be no 3-4 student group that has a single woman or a single student from an underrepresented racial group.

This matching process, albeit time-consuming, leads to a fair balance between logistics and student interests. Generally students are satisfied with their project matches and their groups. Except in extremely rare circumstances, we do not allow students to switch groups or projects. If students are dissatisfied with their project topic, we remind them to keep an open mind (how do they know if they like it or not if they don't know anything about it yet?) and that if they really dislike it, it is only one academic year. To head off group dynamics issues, we explicitly include content within the course to help students understand how to work together successfully, with a specific focus on diverse teams. When group dynamics problems arise, the central mentoring team works intensively with the team and/or faculty mentor to help them resolve their issues.

\subsection{Mentoring Structures}

All ERSP implementations except the 1-quarter Stanford version use the dual-mentoring structure described in Section 3 where the central mentor meets weekly with all ERSP teams to provide high-level guidance, while technical mentors meet weekly with their own team to provide technical support. In the one-quarter Stanford version, graduate TAs meet twice a week with their teams to provide both technical mentorship and high-level guidance.

Some programs have experimented with adding near-peer mentoring to provide additional support. In the second year at UCSD, each ERSP team was assigned an additional mentor from the previous year's ERSP cohort. We found that the near-peer mentors were too busy with their own commitments and did not provide enough proactive guidance to the teams, and thus students did not perceive their value. More recently, we have instead implemented guest panels of past participants in the fall course, and this format has been well-received. In another successful model, at UIC past ERSP participants were hired on an hourly basis as peer mentors for the current year's program. These past participants served as role models, coaches and cheerleaders as teams hit roadblocks. The peer mentors were found to be extremely helpful, not only for ERSP, but for professional and other academic guidance as well.

One of the strengths of the ERSP program is that the dual mentoring structure lifts some of the responsibilities from the technical mentor. Thus ERSP requires minimal technical mentor training (e.g. a single introductory session, plus monthly communication via email) to run successfully. Nevertheless, there is a need for some care in technical mentor selection and to ensure that mentors meet minimal standards and expectations including meeting regularly with students, providing technical support related to the project, and welcoming them into their research groups.

\subsection{Eligibility, Application and Selection}

Students typically apply to ERSP in the spring (current students) or summer (incoming transfer students) prior to the start of the program. Preference goes to students who, at the time of application, have not taken and are not enrolled in any upper division computing courses, though some programs make exceptions on a case-by-case basis. Both current first-year students and incoming junior transfer students are eligible to apply.

The application and review process in all implementations is designed to construct student cohorts with high participation from groups that are currently underrepresented in computing, including women and non-binary students, Black, Latinx and/or Native American students, LGBTQ+ students, and first-generation college students. We also aim to construct a supportive cohort where all participants will feel valued and comfortable, so that students from all groups will have a positive experience.

To achieve these goals, UCSD, UCSB and UIC review student applications based only on their responses to three essay questions:

(1) What do you hope to get out of participating in ERSP?

(2) Explain your understanding of how being from a minoritized group affects a person's experience in CS and how you came about this understanding.

(3) How would your participation in ERSP help increase the diversity of students in the CS community?

Stanford includes the first two questions in the ERSP application.

Each student application is assigned a motivation score (Question 1) and a diversity score (Questions 2 and 3), averaged across two reviewers. The first reviewer is a graduate student or faculty member who is familiar with ERSP and its goals (e.g., someone who has participated in the past); the second is the ERSP program director. Faculty reviewers are provided with a detailed rubric to help them understand how to assign each score. The average diversity score is the primary factor in determining who is admitted, while the average motivation score provides finer-grained distinctions. 


\section{RESOURCES, COSTS, AND COMPENSATION}

Our existing ERSP programs operate at the department level. While broader school- or university-level programs tend to engage only a few students from each department, ERSP engages a large number of students within a single department to build a critical mass of diverse undergraduate researchers. Even at UIC, where the program is implemented in two departments (CS and ECE), a faculty director from each department ensures the program operates on the department level. Once the program is established within a single department, it can be implemented more broadly in additional departments, while still maintaining high participation levels to build a community in each local context.

Budget and Compensation. To run a moderately sized ERSP program (15+ students) requires financial resources at the level of approximately $\$ 1,500$ per student per year to support the critical program elements: the graduate student central mentor, the faculty director, social events, and a poster presentation session. The most critical piece of the program, the graduate student central mentor, also constitutes the largest expense, which varies by the size of the program. We have found that for every 10 hours per week they are hired, the central mentor can manage 6 -7 teams. The second largest expense is support for the ERSP program director. Again, this depends on the size of the program (and the program director's salary!) but we typically budget between $1 / 2$ to 1 month of summer salary. The remainder of the "required" expenses include support for an end of year poster session where students present their work, end-of-program certificate printing, and social activities throughout the year including an end-of-program dinner. Finally, a hidden budget item not included in the amount above is the Introduction to Research course, where the instructor and TA (typically the program director and ERSP central mentor, respectively) must be compensated.

Other items that improve the students' experience in the program are money for supplies to do the research (e.g., books, software, machines, etc.) and money for students to attend conferences, either to present their research or to learn more about the field. These expenses can reasonably be shared with the faculty running the research, but we typically budget some money to assist faculty who may not have the budget to cover these costs or who may be unwilling to spend their money on early undergraduate research.

We often get questions about how to run ERSP in small departments that do not have graduate students available, nor financial resources to support them for a program like this. In these cases, it is still possible to run a small version of ERSP using only faculty resources and very little funding, though it may require faculty to donate their time (which we recognize is not a sustainable or equitable practice), and/or to receive service credit instead of money. A faculty member must be available to teach the Introduction to Research course, but if the program is very small the course might count for only a fraction of a teaching credit, or may initially even be taught on top of the faculty member's normal load (though we do not recommend teaching for free as a long-term solution!). With only $2-3$ teams, a faculty member can serve as both director and central mentor, receiving service credit for their efforts.

All of our programs had some initial seed funding from external sources, but our goal is to provide sufficient guidance that other institutions would be willing to launch the program using internal funds. At UCSD the program is now supported by the CS department who sees the value in the not only engaging a large number of diverse students in research, but also in supporting a graduate student to gain research mentoring skills. Department support is supplemented by faculty who write ERSP into their Broadening Participation plans in their NSF grants.

Considerations for Student and Instructor Credit. Students receive academic credit (not money) for their research participation in ERSP. This choice was made deliberately: throughout the program, student participants typically must take a full load of courses to make progress toward their degree and do not have time to additionally participate in research on top of this full load. By allowing them to do research for credit, and by making this credit count directly toward their degree, they can free up the time in their course schedule to participate in this research. However, this only works if the credit truly counts toward their degree, so we stress this essential piece of the course structure.

Similarly, faculty who teach the Introduction to Research course must do so as an on-load teaching assignment. Asking faculty to volunteer to teach this course will quickly lead to burn-out, and the quality of the course will suffer.

Securing Departmental Support. In addition to financial support as described above, support from leadership in the department of launch is critical to the success of ERSP. Such additional support can entail ensuring the ERSP director's time and efforts are counted towards tenure and promotion, and providing space for the program's promotion both within and outside of the department.

At UCSB, the program director (who at the time of the launch of the program was a new assistant professor) reached out to several senior colleagues to support the program by serving as research mentors. Their support and the decision to start small was critical to launching the program quickly (within 3 weeks from the time funding became available). In the following year, the program director gave a talk about ERSP to colleagues at the annual faculty retreat to share some of the initial outcomes of the program and build additional support.

ERSP may also need to coordinate with existing department UREs. At Stanford, most CS faculty already host students as part of the highly competitive department summer URE. Stanford initially designed the one-quarter version of ERSP in 2019-2020 to provide students with the knowledge and experience to subsequently apply to the department URE. To make the existing URE more accessible to students with less research experience, Stanford then created the multi-quarter version of ERSP, so that participating URE students could build their research skills and mentor-peer relationships prior to the summer. Both versions were offered at Stanford in 2020-2021.

\section{OBSERVATIONS AND REFLECTIONS}

In this section we use participation and survey data to briefly reflect on how well ERSP has met its two primary goals as outlined in Section 3. The goal of this paper is not to formally research ERSP's effectiveness; this has been presented elsewhere $[2,4]$. Here we offer more informal evidence of its broad impact on students to help others make the argument that ERSP is worth implementing. 
Table 1: ERSP retention rates (Ret.) and demographics (Wom./NB = Women and Non-Binary; BLNPI = Black, Latinx, Native American and Pacific Islander) at each institution.

\begin{tabular}{l|c|c|c|cc}
\hline Inst. & Duration & Total & Ret. & Wom./NB & BLNPI \\
\hline UCSD & $2014-21$ & 283 & $95 \%$ & 173 & 65 \\
UCSB & $2018-21$ & 48 & $100 \%$ & 25 & 7 \\
UIC & $2019-21$ & 58 & $97 \%$ & 42 & 16 \\
Stanford & $2019-21$ & 75 & $98 \%$ & 30 & 14 \\
\hline
\end{tabular}

ERSP has met its first goal by engaging and retaining a large number of diverse undergraduates across the four universities. Table 1 shows the participation, demographics and in-program retention for ERSP at each institution. Retention rates are computed as the percentage of students who participated in the full duration of the ERSP program (quarter for Stanford; academic year for others).

Next, we summarize students' reflections from end-of-term feedback surveys collected for the 2019-20 and 2020-21 cohorts across all institutions to address ERSP's second goal: creating an inclusive and supportive community around research. Final surveys at UCSD, UCSB, and UIC included the question, "What was the most valuable part of participating in ERSP?" Stanford included the question, "What would you like to say about this course to a student who is considering taking it in the future?". The average response rates for the surveys across all institutions was $71 \%$. Students across all institutions consistently highlighted the same components of the program that helped them feel included and supported: the professional connections they made, the extensive mentoring and peer support, and the hands-on experience with research.

This quote is representative of how students expressed that ERSP helped them make professional connections:

For some students it can be really hard to connect with faculty because professors are so busy and it can be intimidating to talk to them sometimes, so I think this program is great for allowing for those interactions.

Students also spoke to how the program helped them learn in a supported environment, with feedback like the following:

[T] he most valuable part was to get a more hands-on experience with research while experiencing it with other people who are just as new to it as you are. It's a very humbling experience, but... the fact that you aren't struggling by yourself makes it more comforting since it is a learning experience for everyone.

Participants also reported not just developing technical research skills during the program, but also understanding research as a career. One student wrote:

Getting exposed to graduate mentors and professors allowed me to gauge if I would like the environment in graduate school... It made me more confident in interacting with grad and professors in a research setting. Now I see myself going to graduate school as almost a definite.

Student feedback also reveals areas where specific ERSP implementations can be improved. Despite the care taken to find real research projects appropriately scoped for undergraduates with basic computing (or Electrical Engineering) knowledge, students' experience can vary with respect to the specific project or research group environment. One student in Stanford's quarter-long program felt that the 10-week timeline was too short to complete reasonable research. Another felt that their artificial intelligence project required CS knowledge beyond the core lower-division CS coursework. UCSD has also seen this phenomenon with machine learning (ML) projects in particular-most likely because the ML research often requires extensive math knowledge and familiarity with ML frameworks. In Stanford's quarter-long version, a graduate TA proposes multiple projects in their area of expertise; while the TA is better positioned to provide technical advice, the project context could be inherently inaccessible to ERSP students. In the future, Stanford plans to include a formal ML model as part of the formal course (which UCSD previously added in response to student feedback) and to solicit project ideas from the larger graduate student body to prioritize projects that our students can quickly understand.

Student surveys also reveal some inherent limitations of the ERSP structure. One theme that consistently arises at UCSD in response to the question "What suggestions do you have about how ERSP could be improved?" is that students want more direct instruction of the content they need to learn for their research project. Unfortunately, it is impossible to meet the needs of all groups given that the projects are so different. Another theme is that some students wish they could get started with their project earlier. They find the process of background reading and learning somewhat tedious in the fall and they are eager to get to the "real research." Even at the end of the program, some students seem to lack a bit of perspective that unlike a course assignment, research is not just development, and this background reading and learning is a critical part of the research process.

More broadly, it is unclear to what degree ERSP is succeeding in changing the culture of research at these four institutions to be more inclusive. When we started ERSP, we hypothesized that by bringing a large number of early-career students who do not necessarily fit the mold of traditional "superstar" researchers, we could change faculty perceptions about who "belongs" in research, and ultimately change the research culture from the bottom-up. It is not clear that we have yet achieved this goal. As this project matures, we will explore how to more deliberately address culture change by more explicitly addressing faculty perceptions and practices.

\section{CONCLUSION}

We have presented details of the ERSP model, a program for early undergraduate research in computing that has been implemented at four universities (so far). Our hope is that by sharing our experience, universities can use this paper as a practical guide to implementing the program in their own institution. We have an active and expanding ERSP community who are passionate about building inclusive opportunities for marginalized students to engage in CS research. With this report, we also hope to attract others to contact us and join our community.

\section{ACKNOWLEDGEMENTS}

We would like to thank the ERSP participants for agreeing to allowing their experiences to be studied. We would also like to thank all those who have helped us run ERSP over the years, and Burçin 
Tamer who has helped us evaluate the program. This material is based upon work supported by the National Science Foundation under Collaborative Grant Nos. 1821521, 1821415, 1821449, 1821501.

\section{REFERENCES}

[1] ML Aikens, MM Robertson, S Sadselia, K Watkins, M Evans, CR Runyon, LT Eby, and EL Dolan. 2017. Race and gender differences in undergraduate research mentoring structures and research outcomes. CBE - Life Sciences Education 16 (2017), ar34. http://www.lifescied.org/content/16/2/ar34.full.pdf + html

[2] Christine Alvarado, Alistair Gray, Diba Mirza, and Madeline Tjoa. 2021. The Role of Mentoring in a Dual-Mentored Scalable CS Research Program. Association for Computing Machinery, New York, NY, USA, 945-951. https://doi-org.stanford. idm.oclc.org/10.1145/3408877.3432364

[3] Christine Alvarado, Michael Hilton, Amy J. Ko, Lori Pollock, Kelly Shaw, and Neil Spring. 2020. Best Practices for Expanding Pathways to Undergraduate CS Research Technical Report. Computing Research Association - Education Committee (CRA-E).

[4] Christine Alvarado, Sergio Villazon, and Burçin Tamer. 2019. Evaluating a Scalable Program for Undergraduate CS Research. In Proceedings of the 2019 ACM Conference on International Computing Education Research (Toronto ON, Canada) (ICER '19). Association for Computing Machinery, New York, NY, USA, 269-277. https://doi.org/10.1145/3291279.3339406

[5] L. Barker. 2009. Student and Faculty Perceptions of Undergraduate Research Experiences in Computing. Trans. Comput. Educ. 9, 1, Article 5 (March 2009), 28 pages. https://doi.org/10.1145/1513593.1513598

[6] Michael Barrow, Shelby Thomas, and Christine Alvarado. 2016. ERSP: A Structured CS Research Program for Early-College Students. In Proceedings of the 2016 ACM Conference on Innovation and Technology in Computer Science Education (Arequipa, Peru) (ITiCSE '16). Association for Computing Machinery, New York, NY, USA, 148-153. https://doi.org/10.1145/2899415.2899436

[7] Bethany Bowling, Heather Bullen, Maureen Doyle, and John Filaseta. 2013. Retention of STEM Majors Using Early Undergraduate Research Experiences. In Proceeding of the 44th ACM Technical Symposium on Computer Science Education (Denver, Colorado, USA) (SIGCSE '13). Association for Computing Machinery, New York, NY, USA, 171-176. https://doi.org/10.1145/2445196.2445249

[8] Ben Jelen, Julia Dunbar, Susan Monsey, Olivia K. Richards, and Katie A. Siek. 2019. Utilizing the Affinity Research Group Model in a Summer Research Experience for Undergraduates Program. In Proceedings of the 50th ACM Technical Symposium on Computer Science Education (Minneapolis, MN, USA) (SIGCSE '19). Association for Computing Machinery, New York, NY, USA, 990-996. https://doi.org/10 $1145 / 3287324.3287501$

[9] National Academies of Sciences, Engineering, and Medicine. 2017. Undergraduate Research Experiences for STEM Students: Successes, Challenges, and Opportunities. The National Academies Press, Washington, DC. https://doi.org/10.17226/24622

[10] Christine Pfund, Angela Byars-Winston, Janet Branchaw, Sylvia Hurtado, and Kevin Eagan. 2016. Defining Attributes and Metrics of Effective Research
Mentoring Relationships. AIDS and Behavior 20, S2 (April 2016), 238-248. https://doi.org/10.1007/s10461-016-1384-z

[11] Audrey Smith Rorrer, Joseph Allen, and Huifang Zuo. 2018. A National Study of Undergraduate Research Experiences in Computing: Implications for Culturally Relevant Pedagogy. In Proceedings of the 49th ACM Technical Symposium on Computer Science Education (Baltimore, Maryland, USA) (SIGCSE '18). Association for Computing Machinery, New York, NY, USA, 604-609. https://doi.org/10. $1145 / 3159450.3159510$

[12] Monique Ross, Elizabeth Litzler, and Jake Lopez. 2021. Meeting Students Where They Are: A Virtual Computer Science Education Research (CSER) Experience for Undergraduates (REU). Association for Computing Machinery, New York, NY, USA, 309-314. https://doi.org/10.1145/3408877.3432547

[13] Susan H. Russell, Mary P. Hancock, and James McCullough. 2007. THE PIPELINE: Benefits of Undergraduate Research Experiences. Science 316, 5824 (2007), 548549 .

[14] E. Seymour, A.-B. Hunter, S. L. Laursen, and T. Deantoni. 2004. Establishing the benefits of research experiences for undergraduates in the sciences: First findings from a three-year study. Science Education 88 (2004), 493-534.

[15] Jenny Olin Shanahan, Elizabeth Ackley-Holbrook, Eric Hall, Kearsley Stewart, and Helen Walkington. 2015. Ten Salient Practices of Undergraduate Research Mentors: A Review of the Literature. Mentoring \& Tutoring: Partnership in Learning 23, 5 (2015), 359-376. https://doi.org/10.1080/13611267.2015.1126162 arXiv:https://doi.org/10.1080/13611267.2015.1126162

[16] Jane G. Stout, N. Burçin Tamer, and Christine J. Alvarado. 2018. Formal Research Experiences for First Year Students: A Key to Greater Diversity in Computing? In Proceedings of the 49th ACM Technical Symposium on Computer Science Education (Baltimore, Maryland, USA) (SIGCSE '18). ACM, New York, NY, USA, 693-698. https://doi.org/10.1145/3159450.3159472

[17] Burçin Tamer and Jane G. Stout. 2016. Understanding How Research Experiences for Undergraduate Students May Foster Diversity in the Professorate. In Proceedings of the 47th ACM Technical Symposium on Computing Science Education (Memphis, Tennessee, USA) (SIGCSE '16). ACM, New York, NY, USA, 114-119. https://doi.org/10.1145/2839509.2844573

[18] Heather Thiry and Sandra Laursen. 2011. The Role of Student-Advisor Interactions in Apprenticing Undergraduate Researchers into a Scientific Community of Practice. Journal of Science Education and Technology 20 (12 2011), 771-784. https://doi.org/10.1007/s10956-010-9271-2

[19] Elsa Q. Villa, Kerrie Kephart, Ann Q. Gates, Heather Thiry, and Sarah Hug. 2013. Affinity Research Groups in Practice: Apprenticing Students in Research. Fournal of Engineering Education 102, 3 (2013), 444-466. https://doi.org/10.1002/jee.20016 arXiv:https://onlinelibrary.wiley.com/doi/pdf/10.1002/jee.20016

[20] Helen Walkington, Kearsley A. Stewart, Eric E. Hall, Elizabeth Ackley, and Jenny Olin Shanahan. 2020. Salient practices of award-winning undergraduate research mentors-balancing freedom and control to achieve excellence. Studies in Higher Education 45, 7 (2020), 1519-1532. https://doi.org/10.1080/03075079. 2019.1637838 arXiv:https://doi.org/10.1080/03075079.2019.1637838 